$$
(w, w)-2(u, w)_{s} \leqq(v, v)-2(u, v)_{s} .
$$

Taking (5) and (15) into account, this result may be stated as follows: the functional

$$
\int_{R}\left[\mu v_{i, j} v_{i, j}+(\lambda+\mu) v_{i, i} v_{i, j}\right] d R-2 \int_{S} w_{i}\left[\mu v_{i, j} n_{i}+(\lambda+\mu) v_{i, j} n_{i}\right] d S,
$$

is minimized, over the class of all vectors $v$ satisfying (14), by $w+c$, where $w$ is the solution of (2) and $c$ is any constant vector.

\title{
ON SOME SINGULAR SOLUTIONS OF THE TRICOMI EQUATION*
}

BY G. F. CARRIER AND F. E. EHLERS (Brown University)

1. Introduction. In this note we intend to develop briefly some singular solutions of the Tricomi equation. These solutions have application in the hodograph techniques for the theory of compressible fluids. The flows to which they apply will be discussed in a later work but it is felt that the singular solutions are of sufficient interest to merit this presentation.

2. The singular solutions. We shall consider the equations

$$
\begin{aligned}
\varphi_{x} & =\psi_{y}, \\
\psi_{x} & =-\varphi_{y} / y
\end{aligned}
$$

which imply

$$
\psi_{y y}+y \psi_{x x}=0
$$

and

$$
\varphi_{x x}+\left(\varphi_{y} / y\right)_{y}=0 .
$$

Equation (3) is the Tricomi equation. The solution of primary interest has the following properties:

$$
\begin{aligned}
& \psi_{x} \sim 1 / y \quad \text { on } x=0, y \rightarrow 0 \\
& \varphi_{y} \equiv 0 \quad \text { on } y=0, \text { for } x \neq 0 \\
& \psi, \varphi, \text { regular in } y>0 \text { and on } x=0, y \neq 0 .
\end{aligned}
$$

It is evident that if $\varphi, \psi$, are not regular in $y<0$, the branch lines will occur along the characteristics $x^{2}+4 y^{3} / 9=0$. The development that follows is strictly formal and the proof that the solutions are those sought is readily found by substituting them into the original equations. To find them, we first replace $y$ and $\varphi$ by

$$
s=(2 / 3) y^{3 / 2}, \quad \varphi=(2 / 3)^{2 / 3} y \chi(x, s)=s^{2 / 3} \chi(x, s) .
$$

*Received May 14, 1948. 
Equation (4) becomes

$$
\left(s \chi_{s}\right)_{s}-4 \chi / 9 s+s \chi_{x x}=0 .
$$

We may obtain a solution valid in the elliptic domain $(y>0)$ by the classical Fourier transform technique. We define

$$
\bar{\chi}(\xi, \alpha)=\int_{-\infty}^{\infty} \int_{0}^{\infty} s J_{2 / 3}(\alpha s) e^{-i \xi x} \chi(x, s) d s d x
$$

and multiply Eq. (5) through by $J_{2 / 3}(\alpha s) e^{-i \xi x}$. Integration over the domain $y>0$ of this equation can be carried out as follows. Integration by parts with regard to $s$ yields contributions including some functions of $x$ arising from the boundary condition on $y=0$. The only admissible contribution of this sort is a delta function at the origin, i.e., $\delta(x-0)$. Subsequent integration over $x$ transforms Eq. (5) into the form

$$
\left(\xi^{2}+\alpha^{2}\right) \bar{\chi}=1 \text {. }
$$

However, as implied by Eq. (6),

$$
\chi=\frac{1}{4 \pi^{2}} \int_{0}^{\infty} \int_{-\infty}^{\infty} e^{i \xi x} J_{2 / 3}(\alpha s) \bar{\chi}(\xi, \alpha) d \alpha d \xi
$$

which reduces readily to

$$
\chi(x, s)=C \int_{0}^{\infty} e^{-\alpha|x|} J_{2 / 3}(\alpha s) d \alpha, \quad C \text { being a constant. }
$$

It is also evident that $\int^{x} \chi d x$ or $\chi_{x}$ is a solution of Eq. (5) and, in fact, it can be verified by a simple substitution that

$$
\chi^{(\mu)}(x, s)=\int_{0}^{\infty} \alpha^{\mu-1} e^{-\alpha|x|} J_{2 / 3}(\alpha s) d \alpha
$$

is a solution. However ${ }^{1}$, the integral of Eq. (10) is (when it exists)

$$
\chi^{(\mu)}(x, s)=\frac{(s / 2)^{2 / 3} \Gamma(\mu+2 / 3)}{\Gamma(5 / 3)\left(x^{2}+s^{2}\right)^{\mu / 2+1 / 3}} F\left(\frac{3 \mu+2}{6}, \frac{4-3 \mu}{6} ; \frac{5}{3} ; \frac{s^{2}}{x^{2}+s^{2}}\right) .
$$

It is evident that (in Eq. 8) we might have used $J_{-2 / 3}(\alpha s)$ instead of the Bessel function of order $2 / 3$. In this case

$$
\chi^{\left(\mu^{\prime}\right)}(x, s)=\frac{(s / 2)^{-2 / 3} \Gamma(\mu-2 / 3)}{\Gamma(1 / 3)\left(x^{2}+s^{2}\right)^{\mu / 2-1 / 3}} F\left(\frac{3 \mu-2}{6}, \frac{1-3 \mu}{6} ; \frac{1}{3} ; \frac{s^{2}}{x^{2}+s^{2}}\right) .
$$

It is now readily shown that a linear combination of the functions defined by Eqs. (11), (12), is the desired solution provided $\mu=0$. This again is a formal step since the integral which leads to Eq. (12) does not converge for $\mu=0$. However, by arguments involving analytic continuation (or again by the trivial substitution) the functions of (11) and (12) can be seen to constitute proper solutions for all $\mu$.

For $\varphi^{(\mu)}$ we choose the combination

$$
\varphi^{(\mu)}=A(\mu) s^{2 / 3} \chi^{(\mu)}+B(\mu) s^{2 / 3} \chi^{\left(\mu^{\prime}\right)},
$$

'See G. N. Watson, Bessel functions, Macmillan Company, New York, 1944, p. 385. 
where

$$
\begin{aligned}
& A(\mu)=\frac{\Gamma(1 / 3) \Gamma(2 / 3) \Gamma(1 / 2)}{\Gamma(\mu-2 / 3) \Gamma(\mu+1 / 3) \Gamma(5 / 6-\mu / 2)}, \\
& B(\mu)=\frac{\Gamma(-2 / 3) \Gamma(5 / 3) \Gamma(1 / 2)}{\Gamma(1 / 6-\mu / 2) \Gamma(\mu / 2-1 / 3) \Gamma(\mu+2 / 3)} .
\end{aligned}
$$

Any other combination is not regular in a sufficiently general region. With the aid of an identity $^{2}$ for the hypergeometric functions and of Eq. (11) and (12), this reduces to the following equation for $\mu=0$

$$
\varphi=\left(x^{2}+s^{2}\right)^{1 / 3} F\left(-\frac{1}{3}, \frac{1}{6} ; \frac{1}{2} ; \frac{x^{2}}{x^{2}+s^{2}}\right) .
$$

This is the desired solution for $\varphi$.

Retaining the integrals in Eq. (13), we compute the conjugate function $\psi$ for arbitrary $y$. Since

$$
\psi_{x}=-(1 / y)(\partial \varphi / \partial y)=-\left(y^{-1 / 2}\right)(\partial \varphi / \partial s)=-(2 / 3)^{1 / 3} s^{-1 / 3} \partial \varphi / \partial s
$$

we obtain

$$
\begin{aligned}
(3 / 2)^{1 / 3} \psi=2^{-2 / 3} A(\mu) s^{1 / 3} \int_{0}^{\infty} e^{-|x| \alpha} J_{1 / 3}(\alpha s) \alpha^{\mu-1} d \alpha \\
-2^{2 / 3} B(\mu) s^{1 / 3} \int_{0}^{\infty} e^{-|x| \alpha} J_{-1 / 3}(\alpha s) \alpha^{\mu-1} d \alpha .
\end{aligned}
$$

Replacing the integrals and simplifying the coefficients leads to

$$
\begin{aligned}
\psi=\frac{4^{1 / 3}}{3} & \frac{y}{\left(x^{2}+4 y^{3} / 9\right)^{1 / 6}} F\left(\frac{1}{6}, \frac{2}{3} ; \frac{4}{3} ; \frac{4 y^{3} / 9}{x^{2}+4 y^{3} / 9}\right) \\
& -(4 / 3)^{1 / 3}\left(x^{2}+4 y^{2} / 9\right)^{1 / 6} F\left(-\frac{1}{6}, \frac{1}{3} ; \frac{2}{3} ; \frac{4 y^{3} / 9}{x^{2}+4 y^{3} / 9}\right)
\end{aligned}
$$

for $y=0$. It is easily verified that (14) and (15) define functions having the desired properties.

One should note that these functions can be taken as single valued except in the domain $\left(x^{2}+s^{2}\right)<0$. In this region they are essentially triple valued and, in fact, this is precisely the property required for the application hinted at in the introduction. ${ }^{3}$ We might add that a more general solution regular in $x^{2}+s^{2}>0$, multi-valued in $x^{2}+$ $s^{2}<0$, and having the behavior $\varphi_{y} \sim y^{3 v-1}$ as $y \rightarrow 0, x \equiv 0$, is

$$
\varphi=\left(x^{2}+s^{2}\right)^{\nu} F\left(-\nu, \nu-\frac{1}{6} ; \frac{1}{2} ; x^{2} /\left(x^{2}+s^{2}\right)\right) .
$$

It seems worth while to also record another singular solution of interest. It is the

${ }^{2}$ E. T. Whitaker and G. N. Watson, Modern analysis, University Press, Cambridge, 1940, p. 291.

${ }^{3} \mathrm{~A}$ discussion of the necessity of this property can be found in Lighthill, M. J., The hodograph transformation in trans-sonic flow. I. Symmetrical channels, Proc. Roy. Soc. London (A) 191, 323 (1947). 
solution such that $\varphi$ has a logarithmic singularity at the point $x_{0}, y_{0} ; \psi$ (of course) has an "arc tan behavior" at this point. It is immediately evident that we are looking for the Green's function of Eq. (5), say for the region $y>0$. To find this, we merely put a spurious. non-homogeneous term $N(x, s)$ on the right side of (5), define $\bar{N}$ as we did $\bar{\chi}$, and operate as before.

This time we find

$$
\left(\xi^{2}+\alpha^{2}\right) \bar{\chi}=\bar{N}
$$

if we allow the boundary contributions to vanish. The inversion of Eq. (17) leads to

$$
\chi=\int_{-\infty}^{\infty} \int_{0}^{\infty} s_{0} N\left(x_{0}, s_{0}\right) \int_{-\infty}^{\infty} \int_{0}^{\infty} \frac{\alpha e^{i \xi\left(x-x_{0}\right)} J_{2 / 3}(\alpha s)}{\xi^{2}+\alpha^{2}} J_{2 / 3}\left(\alpha s_{0}\right) d \xi d \alpha d x_{0} d s_{0}
$$

where the double integral over $\xi$ and $\alpha$ must define the Green's function. Thus, we write

$$
\begin{aligned}
G\left(x, x_{0} ; s, s_{0}\right) & =\int_{0}^{\infty} e^{-\alpha\left|x-x_{0}\right|} J_{2 / 3}(\alpha s) J_{2 / 3}\left(\alpha s_{0}\right) d \alpha \\
& =A s^{-1 / 2} Q_{1 / 6}\left(\frac{\left(x-x_{0}\right)^{2}+s^{2}+s_{0}^{2}}{2 s s_{0}}\right)
\end{aligned}
$$

where $A$ is some constant.

The corresponding solutions $\varphi$ and $\psi$ are

$$
\begin{aligned}
& \varphi=y^{1 / 4} Q_{1 / 6}\left(\frac{\left(x-x_{0}\right)^{2}+y^{3}+y_{0}^{3}}{2\left(y y_{0}\right)^{3 / 2}}\right) \\
& \psi=\int^{x}\left(\varphi_{\nu} / y\right) d x
\end{aligned}
$$

It is interesting to note that the stream function $\psi$ is closely related to one found by Weinstein. ${ }^{4}$

If we had formally used $J_{-2 / 3}$ again, the index $1 / 6$ appearing in the Legendre function would be replaced by $-7 / 6$. Either solution may readily be continued into the hyperbolic domain.

\section{THE LOST SOLUTIONS IN AXIALLY SYMMETRIC IRROTATIONAL COMPRESSIBLE FLUID FLOW*}

\section{By H. J. STEWART (California Institute of Technology)}

1. Since the two dimensional potential equation for the irrotational flow of a compressible fluid may be linearized by a Legendre transformation from the physical plane to the velocity (hodograph) plane, this linearizing transformation is usually one of the first steps taken in the study of two dimensional compressible fluid flows. This trans-

${ }^{4}$ Weinstein, A., On axially symmetric flows, Q. Appl. Math. 5, 429 (1948).

*Received Jan. 12, 1948. 